\title{
Dynamic Spectra of Small-Mass Meteors
}

\author{
Emma R. Mirizio ${ }^{1}$, Robert G. Michell ${ }^{2}$, and Marilia Samara ${ }^{2}$ \\ ${ }^{1}$ University of Maryland, College Park, MD, USA \\ ${ }^{2}$ NASA Goddard Space Flight Center, Greenbelt, MD, USA \\ Correspondence: Emma R. Mirizio (emmamirizio@gmail.com)
}

\begin{abstract}
We present dynamic (22 frames per second) observations of optical spectra of small-mass (2-200 mg) meteors observed with an EMCCD imager equipped with a diffraction grating. This observational campaign occurred at Arecibo, Puerto Rico during May 2012, resulting in eight hours of clear data over four nights. We detected 22 meteors with this setup and their spectra showed varying compositions, including evidence of $\mathrm{Na}, \mathrm{Mg}, \mathrm{Fe}$, and $\mathrm{Ca}$. Spectral lines persisting over multiple frames (up 23 frames) with sufficient signal, showed evidence for differential ablation. Brighter, more massive meteors had stronger and varied spectral signals, which showed that the temporal and spectral resolution of the faintest meteors approached the noise level of the camera system. Optical and spectral detections of these small-mass meteors provide a greater understanding of the composition of the milligram-sized population of meteors.
\end{abstract}

\section{Introduction}

Small-mass meteoroids and cosmic dust particles are deposited into Earth's atmosphere when their orbits intersect Earth. This material is a direct result of collisions in the asteroid belt and the sublimation of materials in comets as they approach the sun. When these meteoroids ablate in the upper atmosphere, they deposit various metals which can often coalesce into distinct metal layers within the atmosphere (Vondrak et al., 2008). Various detection methods have been used to place the total amount 15 of all meteoric material entering the atmosphere at a range from just 5 tons up to 300 tons of material per day (Plane, 2012). This vast discrepancy has drastic implications on the composition of the upper atmosphere. First, the composition of metal and ion layers in the mesosphere (altitudes of 80 to $105 \mathrm{~km}$ ) is determined by the total amount of material that ablates and ionizes to create these structures. In addition, particles and metals in the atmosphere can be used as markers for climate change (Plane, 2012). A better understanding of the total amount and composition of meteoric material ionized in the atmosphere is needed in order to better constrain global circulation models of Earth's atmosphere.

Meteor mass estimates are found by two main methods-radar observations and optical observations. In general, radar observations are best at detecting the more numerous smaller mass meteors (Mathews et al., 2001; Close et al., 2005; Dyrud and Janches, 2008; Fentzke and Janches, 2008; Close et al., 2012; Janches et al., 2014b; Michell et al., 2019), while optical observations are better suited to observing the less numerous, larger mass (optically brighter) meteor population. Both of these 

initial meteoroid mass from observable parameters. Radar observations use an assumption about the ionization efficiency, which is essentially the number of free electrons generated per initial meteoric atom. (Campbell-Brown et al., 2012) gives a more complete description of ionization efficiencies including how they are modeled and estimated. Optical observations rely on an assumption about how much of the meteors initial kinetic energy gets converted into photon energy, which is called the luminous efficiency (McKinley, 1961; Campbell-Brown et al., 2012).

Radar observations to find meteor head-echoes are performed using High-Power and Large Aperture (HPLA) radar, also called Incoherent Scatter Radar (ISR). These are used to determine the flux of a large number of milligram and microgram sized meteors and dust particles, for example (Mathews et al., 2001; Close et al., 2005; Dyrud and Janches, 2008; Fentzke and Janches, 2008; Close et al., 2012; Janches et al., 2014b). This method can be used to determine the line-of-sight velocity as well as the deceleration along the meteor's path, as well as the backscattered power from the meteor. However, it can only be used to measure a lower limit for the total velocity unless the radar is using interferometry (Sato et al., 2000; Chau and Woodman, 2004; Sparks et al., 2010; Janches et al., 2014a).

Optical observations of the sporadic meteor background typically detect larger and brighter meteors in the milligram to gram range, but sacrificing temporal or spatial resolution allows this detection method to observe very faint meteors. The 40 combination of optical and radar data provides a more accurate constraint on meteoric properties such as the total velocity and originating radiant direction. Estimates of meteoroid mass have been made by this method as shown in (Campbell-Brown et al., 2012; Weryk and Brown, 2013; Brown et al., 2017; Michell et al., 2019).

Optical spectroscopy can be used to determine the composition of meteors which could enable even better mass estimates to be made, because the ablation process and composition would be better understood and therefore less assumptions would need to be made in order to calculate the meteoroid mass. Spectroscopic observations of meteors have been thoroughly studied for larger mass meteors, or fireballs (Ceplecha, 1971; Borovicka, 1993; Trigo-Rodriguez et al., 2003), and in meteor showers (Millman, 1963). In addition, all-sky cameras have similarly been used to find greater numbers of fireballs and their spectra (Rudawska et al., 2016). Meteor composition for small mass meteors have been estimated from meteor head-echoes for microgram sized meteors in the sporadic background (Janches et al., 2009). (Jenniskens, 2004) explored the detection and

50 composition of milligram sized fast meteors within the Leonid shower using an aircraft with attached spectrographs and optical cameras to detect milligram sized meteors.

Small-mass meteors in the sporadic background are more difficult to study optically, because they have faint optical signals, requiring telescopic (small) fields of view and thus the meteors occur less frequently within the imager FOV, requiring longer observation periods. Meteor showers have the advantage that a larger number of meteors can occur in a relatively short time,

55 thus requiring shorter overall observing times. Spectroscopy can give insight into relative abundances of each element within a meteor, and be used to detect differential ablation when a meteor is observed over a period of time. Common elements in sporadic meteors include $\mathrm{Mg}, \mathrm{Fe}, \mathrm{Ni}$, and $\mathrm{Na}$ as well as smaller abundances of $\mathrm{Ca}$ (Harvey, 1973). Ablation of small-mass meteors and dust particles has been modeled to confirm differential ablation and relative abundances of ionized particles in the meteors. More volatile elements, $\mathrm{Na}$ and $\mathrm{K}$, ablate earlier, or higher in the atmosphere compared to less volatile elements, Fe, 
$60 \mathrm{Mg}$, and $\mathrm{Si}$, which are all major constituents of meteor composition (Vondrak et al., 2008). Data from the Arecibo radar has shown evidence of this differential ablation process based on meteor altitude and velocity, for example (Janches et al., 2009).

The next section describes the overall methodology used in the following analyses. Then a description of the results and their implications is presented, followed by an overall summary.

\section{Methodology}

\section{$65 \quad 2.1 \quad$ Optical}

The observations presented here are of meteor spectra taken with a small field of view $\left(13^{\circ}\right)$ Electron Multiplying CCD (EMCCD) imager, where meteor spectra were observed for meteors with absolute visual magnitudes down to +3.0 , corresponding to masses down to a few milligrams. These observations were taken during an observational campaign conducted at the Arecibo Observatory in Puerto Rico (18.3 $\left.{ }^{\circ} \mathrm{N}, 66.8^{\circ} \mathrm{W}\right)$ during May 2012. This campaign utilized an Andor Ixon DU-888 EMCCD 70 imager that is part of the Multi-spectral Observatory Of Sensitive EMCCDs (MOOSE) imagers, as described in (Michell et al., 2019). To reduce noise and ensure a signal from faint meteors, the CCD was cooled to $-70 \mathrm{C}$ and binned $2 \times 2$. A short integration time was chosen, which gave 22 frames per second. The $380 \times 380$ pixel frame resulted in an angular resolution of 0.036 degrees per pixel, resulting in a total field-of-view of 13 degrees. The optical camera was pointed in the local zenith at the Arecibo optical site. This campaign produced a total of 8 hours of observations with clear sky without clouds over four nights from May 17-20, 2012 and a total of 22 meteors were detected with measurable spectra.

The diffraction grating used produced a spectral resolution of $\sim 7 \mathrm{~nm}$ per pixel and covered the range from $400 \mathrm{~nm}$ to 800 $\mathrm{nm}$. The spectral calibration was done in the lab prior to the campaign using a Neon-Argon light source with known spectral lines. The diffraction grating was aligned on the imager lens such that the spectra were dispersed in the north-south direction in order to maximize the spectral resolution of the generally east-west traveling meteors.

80 These observations were taken at the same time as the dual radar and optical observations presented in (Michell et al., 2019). The motivation was to be able to increase the accuracy of our mass estimates by measuring the actual spectra of the meteors detected by multiple methods. However, given the reduced sensitivity of the imager with the spectrograph grating and the larger field-of-view, the meteors that were detected with measurable spectra did not have corresponding radar returns because they were either too far away from the radar beams, or passed through the nulls in the beam pattern, while still being inside the imager field-of-view. Therefore no radar data is presented and the focus of this paper is on the properties derived from the optical spectra alone, including finding lower limits on their velocity and upper limits on mass for each meteor as well as their elemental compositions.

Figure 1 shows an example frame from the imager, illustrating a meteor and its spectra (left) and an example image showing stars and their corresponding spectra (right) that were used as calibration sources. The F0 star shown was used for calibrating the wavelength sensitivity of the combined imager-diffraction grating system. 

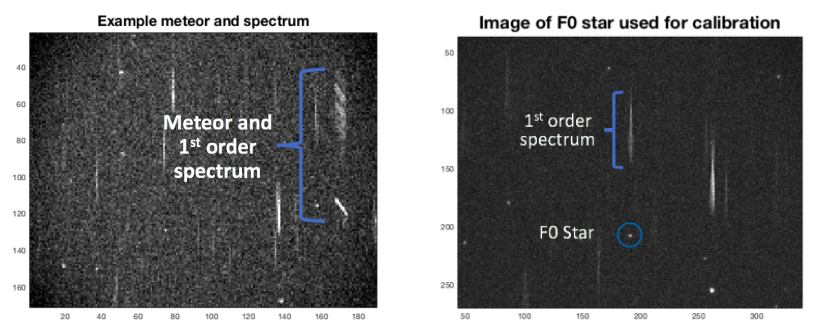

Figure 1. An example raw image of a meteor and its spectra (left) that is uncalibrated and unflattened. An example stellar spectra (right) for a type F0 star is shown. This star was used for calibrating the wavelength sensitivity of the combined imager-diffraction grating system.

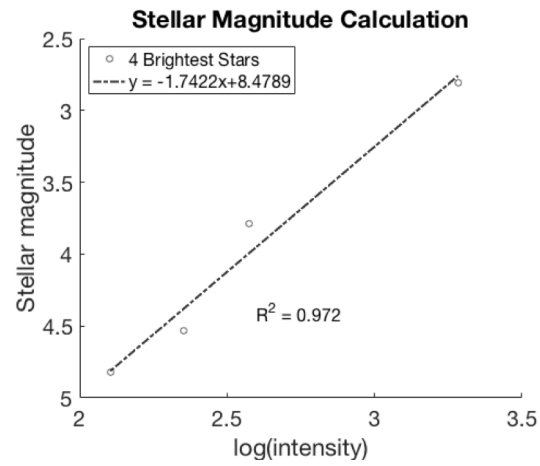

Figure 2. The calibration between the stellar magnitudes and the logarithm of the imager counts. The linear fit values and the $\mathrm{R}^{2}$ values are given in the legend.

\subsection{Stellar Calibration}

The first step in the imager calibration was to use reference stars in the images to convert the intensities from the imager (in counts) into stellar magnitudes. This was accomplished by creating a linear fit between the magnitudes of known reference stars and the logarithm of the intensities (in counts) from the imager. Figure 2 shows this relationship for the four brightest stars in the image, where clear intensities were able to be derived from the zeroth-order spectra (un-diffracted images). For these data, we found a linear fit, with an $\mathrm{r}^{2}$ value of 0.972 using the four brightest stars in an image. This linear fit was then used to convert the imager counts corresponding to the meteors into stellar magnitude values which were then used to calculate the meteor masses, using the kinetic energy and luminosity relationship found in (McKinley, 1961). For the 22 meteors detected, we can place lower bounds on the mass, because we assumed that the only measured component of the velocity (the horizontal component) was the total speed, knowing the the total speed must have been higher. In addition, without radar returns or another imager for triangulation, we simply assumed that the altitude of all the meteors was $95 \mathrm{~km}$, which will also contribute to the uncertainties in the mass estimates.

In order to characterize the relative intensities of the observed emission lines in the meteor spectra, we need to quantify the imager response with the diffraction grating as a function of wavelength (the spectral response), which was not done in the lab 

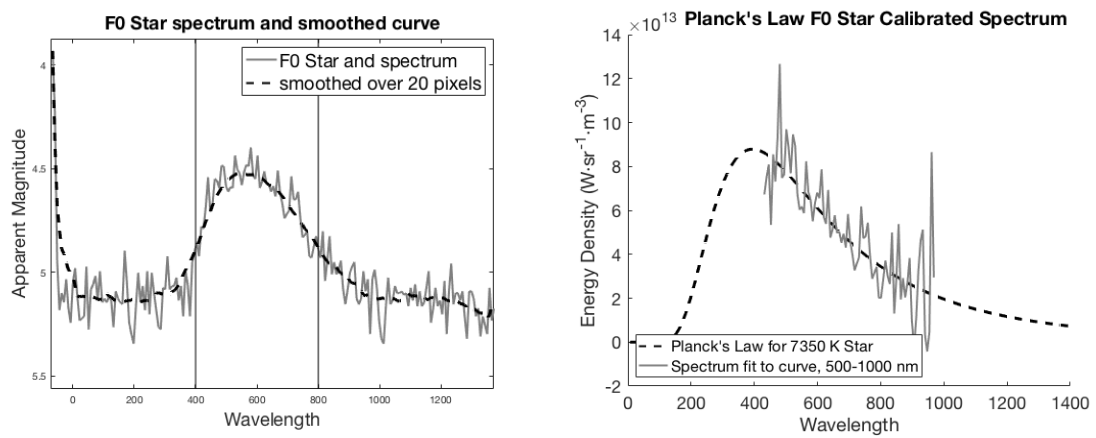

Figure 3. The measured F0 stellar spectrum showed that the spectrum responded between 400 and $800 \mathrm{~nm}$ (left) and this response was fit to a Planck curve with a temperature of $7350 \mathrm{~K}$ (right).

at the time. Due to quantum efficiency effects of the CCD detector itself and the transmission characteristics of all the lenses, there will be a spectral dependence on the measured intensity of the meteor spectra that needs to be normalized out. To do this, a star of a known spectral type (F0 in this case), was examined. Figure 3 shows the measured spectra from an F0 type star, fit to a Planck curve with a temperature of $7350 \mathrm{~K}$. This was then used to create a wavelength normalization that was applied to the meteor spectra, such that the relative intensities of the observed emission lines were of physical significance and not just instrumentally created. Atmospheric transmittance corrections were also applied to each spectrum that affected the shorter wavelengths (350 - $400 \mathrm{~nm})$.

\section{Observations}

There were a total of 22 meteors observed, where spectra were discernible. Table 1 lists a summary of the main characteristics of these 22 meteors, including the date, time in UT, the horizontal velocity in $\mathrm{km} / \mathrm{s}$, the azimuthal direction of origin in degrees where East $=0^{\circ}$ and North $=90^{\circ}$, the maximum brightness in visual magnitude and the derived mass in milligrams. The maximum brightnesses ranged between 3.2 and 0.4 magnitude and the masses ranged between 2.4 and $153.1 \mathrm{mg}$.

As shown in Table 1, strong spectral lines were visible for $\mathrm{Ca}, \mathrm{Fe}, \mathrm{Mg}$, and $\mathrm{Na}$ in some meteors. Lines were observed at 518 $\mathrm{nm}$ and $553 \mathrm{~nm}$ for $\mathrm{Mg}$ (Savage and Boitnott, 1971), and in all cases where Mg was present, both spectral lines were visible. In addition, strong $\mathrm{Na}$ lines were only visible in the brightest meteors that also showed evidence for the other three observed elements. This analysis was done by observing the full evolution of each spectrum, and peaks were made clear by summing over multiple frames.

Figures 4 and 5 show the spectrum of meteor 4. This meteor was visible for 11 frames, or 0.5 seconds, and higher wavelengths of the spectrum were not visible for the first half of the meteor's path across the detector. The Fe line is not visible until more than halfway through the meteor's path, while Mg is seen as an excited line throughout the spectrum. Although part of the spectrum is lost due to the limited field of view, the 518 line of $\mathrm{Mg}$ is seen in both the beginning and end of the spectrum. 
Table 1. Summary of the main characteristics of the 22 meteors detected, showing the meteor number, the date, time in UT, the horizontal velocity in $\mathrm{km} / \mathrm{s}$, the azimuthal direction of origin in degrees where East $=0^{\circ}$ and North $=90^{\circ}$, the maximum brightness in visual magnitude, the derived mass in milligrams, and spectral lines that were discernible.

\begin{tabular}{|c|c|c|c|c|c|c|c|}
\hline \# & Date & Time (UT) & H. Vel. $(\mathrm{km} / \mathrm{s})$ & Azimuth (deg.) & Mag. & Mass (mg) & Elements Found \\
\hline 1 & 17 May & $07: 18: 24$ & 39.6 & $101.0^{\circ}$ & 2.3 & 4.76 & $\mathrm{Ca}, \mathrm{Fe}$ \\
\hline 2 & 17 May & $07: 20: 24$ & 35.3 & $151.1^{\circ}$ & 2.7 & 5.35 & $\mathrm{Ca}$ \\
\hline 3 & 17 May & $07: 25: 06$ & 35.9 & $109.2^{\circ}$ & 2.8 & 4.04 & $\mathrm{Ca}, \mathrm{Fe}$ \\
\hline 4 & 17 May & $07: 25: 16$ & 40.0 & $145.4^{\circ}$ & 2.0 & 12.64 & $\mathrm{Ca}, \mathrm{Mg}, \mathrm{Fe}$ \\
\hline 5 & 17 May & 08:16:01 & 32.2 & $151.9^{\circ}$ & 2.9 & 2.42 & $\mathrm{Ca}, \mathrm{Mg}, \mathrm{Na}$ \\
\hline 6 & 17 May & 08:20:06 & 25.3 & $167.0^{\circ}$ & 2.9 & 7.72 & \\
\hline 7 & 17 May & 08:31:00 & 23.4 & $236.3^{\circ}$ & 2.7 & 46.30 & $\mathrm{Ca}, \mathrm{Mg}, \mathrm{Fe}, \mathrm{Na}$ \\
\hline 8 & 17 May & $08: 37: 30$ & 19.3 & $121.6^{\circ}$ & 2.8 & 12.97 & $\mathrm{Ca}, \mathrm{Mg}, \mathrm{Fe}$ \\
\hline 9 & 17 May & $08: 48: 25$ & 9.0 & $127.4^{\circ}$ & 3.2 & 53.09 & \\
\hline 10 & 17 May & $08: 49: 54$ & 38.0 & $109.9^{\circ}$ & 2.7 & 7.11 & $\mathrm{Ca}$ \\
\hline 11 & 18 May & $05: 54: 06$ & 20.1 & $221.0^{\circ}$ & 3.0 & 11.81 & \\
\hline 12 & 18 May & $06: 12: 18$ & 17.9 & $239.8^{\circ}$ & 1.8 & 153.16 & $\mathrm{Ca}, \mathrm{Mg}, \mathrm{Fe}, \mathrm{Na}$ \\
\hline 13 & 18 May & $06: 53: 51$ & 55.3 & $174.2^{\circ}$ & 1.2 & 11.65 & \\
\hline 14 & 19 May & $01: 35: 59$ & 38.1 & $151.2^{\circ}$ & 1.9 & 23.43 & \\
\hline 15 & 19 May & 01:49:39 & 24.3 & $146.5^{\circ}$ & 1.1 & 84.08 & \\
\hline 16 & 19 May & $08: 14: 23$ & 68.0 & $98.4^{\circ}$ & 1.7 & 3.94 & \\
\hline 17 & 19 May & $08: 18: 10$ & 33.6 & $138.2^{\circ}$ & 2.0 & 7.64 & \\
\hline 18 & 20 May & $07: 41: 50$ & 46.6 & $52.3^{\circ}$ & 1.8 & 10.96 & $\mathrm{Mg}, \mathrm{Fe}, \mathrm{Na}$ \\
\hline 19 & 20 May & $07: 55: 30$ & 23.0 & $346.0^{\circ}$ & 2.1 & 55.66 & $\mathrm{Ca}, \mathrm{Mg}$ \\
\hline 20 & 20 May & 08:04:37 & 62.4 & $107.9^{\circ}$ & 1.4 & 5.50 & $\mathrm{Ca}, \mathrm{Mg}, \mathrm{Na}$ \\
\hline 21 & 20 May & $08: 33: 13$ & 77.0 & $154.1^{\circ}$ & 1.9 & 4.29 & $\mathrm{Mg}, \mathrm{Na}$ \\
\hline 22 & 20 May & $08: 38: 46$ & 62.7 & $123.9^{\circ}$ & 0.4 & 17.57 & $\mathrm{Ca}, \mathrm{Fe}, \mathrm{Na}$ \\
\hline
\end{tabular}

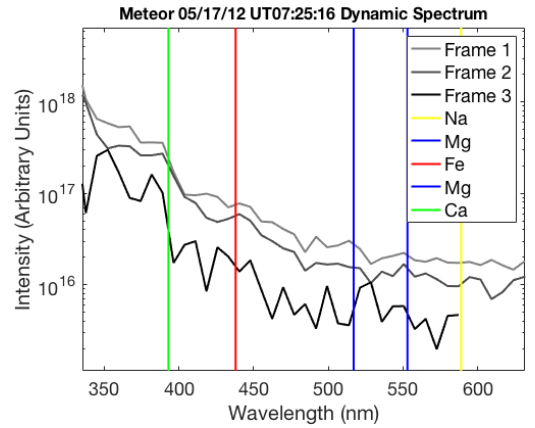

Figure 4. Evolution of a meteor over 6 frames, or 0.3 seconds, showing the Fe spectral line appearing after the Mg line remains visible. 


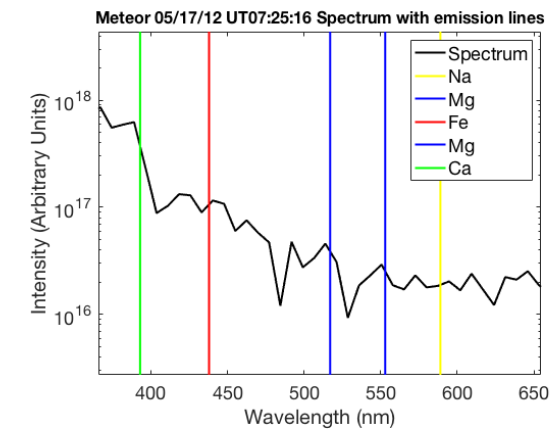

Figure 5. Meteor showing evidence of all elements listed except Na.

\section{Discussion and Conclusions}

Differential ablation has previously been detected at Arecibo using the $430 \mathrm{MHz}$ radar by observing the head-echoes of smallmass meteors (Janches et al., 2009) and similarly detected by the Resolute Bay ISR, another HPLA radar (Malhotra and Mathews, 2011). With radar, compositional differences were determined by measurements made at various altitudes over time. At higher altitudes, more volatile elements are observed, confirming that the differential ablation evidence agrees with the model. Although the optical data lacks altitude measurements, figures 4 and 5 also show more volatile elements are observed at a higher density earlier in time, implying the altitude relationship.

$\mathrm{Ca}$ lines were identified in almost all of the 22 meteors in this campaign, and $\mathrm{Na}, \mathrm{Fe}$, and $\mathrm{Mg}$ were observed in many meteors.

Spectral observations of these meteors come close to the noise level of the camera. The diffraction grating spreads photons from the already optically-faint, small-mass meteors, into a range of wavelengths. This causes the first order spectrum of each meteor to become faint at both extremes of the wavelength spectrum. Nearing the smaller wavelengths $(<500 \mathrm{~nm})$, artifacts can appear from the normalization process. This is due to the increasing intensity of background radiation in the spectra which is visible in the star spectrum in Figure 3. In addition, the short exposure time of the detector ensures good temporal resolution to identify differential ablation, but sacrifices signal in each frame. For the fainter meteors, it was necessary to coadd multiple frames in order to identify the composition.

We presented a study of optical spectra for 22 milligram-size meteors which showed evidence for differential ablation. This result pushes the observational limitations of the current camera system, using a diffraction grating to optically detect very faint meteors at high enough signals to detect spectral lines at high temporal cadence. Stronger and more varied spectral lines were found in meteors with greater brightnesses and therefore greater mass estimates, which shows that the observations of the faintest and smallest meteors approached the noise level of the detector. These observations present a case for the feasibility of using such spectral observations of smaller mass meteors to begin to better characterize the composition of the the smaller mass (milligram-sized) meteors. As the capabilities of imaging technology increases, so will our knowledge of the smaller, more numerous meteors through targeted spectral observations. 
https://doi.org/10.5194/angeo-2020-61

Preprint. Discussion started: 21 September 2020

(c) Author(s) 2020. CC BY 4.0 License.

Data availability. The optical and spectral data of each meteor can be accessed on Github, DOI 10.5281/zenodo.4009858. Raw data and

\section{Appendix A}

\section{A1}

Author contributions. Robert G. Michell and Marilia Samara designed and conducted the experiment. Robert G. Michell also reviewed, edited, and supervised the writing and analysis. Emma R. Mirizio developed software, performed analysis, created visualizations, and prepared the manuscript with contributions from coauthors.

Competing interests. The authors declare that they have no conflict of interest.

Acknowledgements. These observations were funded in part by a Southwest Research Institute internal research and development grant. Robert G. Michell was supported by NSF Grant \# AGS1523097 


\section{References}

160 Borovicka, J.: A fireball spectrum analysis, Astronomy and Astrophysics, 279, 627-645, 1993.

Brown, P., Stober, G., Schult, C., Krzeminski, Z., Cooke, W., and Chau, J.: Simultaneous optical and meteor head echo measurements using the Middle Atmosphere Alomar Radar System (MAARSY): Data collection and preliminary analysis, Planetary and Space Science, 141, 25 - 34, https://doi.org/https://doi.org/10.1016/j.pss.2017.04.013, http://www.sciencedirect.com/science/article/pii/S0032063316304020, 2017.

Campbell-Brown, M. D., Kero, J., Szasz, C., Pellinen-Wannberg, A., and Weryk, R. J.: Photometric and ionization masses of meteors with simultaneous EISCAT UHF radar and intensified video observations, Journal of Geophysical Research (Space Physics), 117, A09323, https://doi.org/10.1029/2012JA017800, 2012.

Ceplecha, Z.: Evidence from Spectra of Bright Fireballs, International Astronomical Union Colloquium, 13, 89-102, https://doi.org/10.1017/S0252921100048971, 1971.

Chau, J. L. and Woodman, R. F.: Observations of meteor-head echoes using the Jicamarca 50MHz radar in interferometer mode, Atmospheric Chemistry \& Physics, 4, 511-521, 2004.

Close, S., Oppenheim, M., Durand, D., and Dyrud, L.: A new method for determining meteoroid mass from head echo data, J. Geophys. Res., 110, 9308, https://doi.org/10.1029/2004JA010950, 2005.

Close, S., Volz, R., Loveland, R., Macdonell, A., Colestock, P., Linscott, I., and Oppenheim, M.: Determining meteoroid bulk densities using a plasma scattering model with high-power large-aperture radar data, ICARUS, 221, 300-309, https://doi.org/10.1016/j.icarus.2012.07.033, 2012.

Dyrud, L. and Janches, D.: Modeling the meteor head echo using Arecibo radar observations, Journal of Atmospheric and Solar-Terrestrial Physics, 70, 1621-1632, https://doi.org/10.1016/j.jastp.2008.06.016, 2008.

Fentzke, J. T. and Janches, D.: A semi-empirical model of the contribution from sporadic meteoroid sources on the meteor input function in the MLT observed at Arecibo, Journal of Geophysical Research (Space Physics), 113, A03304, https://doi.org/10.1029/2007JA012531, 2008.

Harvey, G. A.: Elemental abundance determinations for meteors by spectroscopy, Journal of Geophysical Research (1896-1977), 78, 39133926, https://doi.org/10.1029/JA078i019p03913, https://agupubs.onlinelibrary.wiley.com/doi/abs/10.1029/JA078i019p03913, 1973.

Janches, D., Dyrud, L. P., Broadley, S. L., and Plane, J. M. C.: First observation of micrometeoroid differential ablation in the atmosphere, Geophys. Res. Lett., 36, L06101, https://doi.org/10.1029/2009GL037389, 2009.

Janches, D., Hocking, W., Pifko, S., Hormaechea, J. L., Fritts, D. C., Brunini, C., Michell, R., and Samara, M.: Interferometric meteor head echo observations using the Southern Argentina Agile Meteor Radar, J. Geophys. Res., 119, https://doi.org/doi:10.1002/2013JA019241, 2014a.

Janches, D., Plane, J. M. C., Nesvorný, D., Feng, W., Vokrouhlický, D., and Nicolls, M. J.: Radar Detectability Studies of Slow and Small Zodiacal Dust Cloud Particles. I. The Case of Arecibo 430 MHz Meteor Head Echo Observations, The Astrophysical Journal, 796, 41, https://doi.org/10.1088/0004-637X/796/1/41, 2014b.

Jenniskens, P.: Meteor induced chemistry, ablation products, and dust in the middle and upper atmosphere from optical spectroscopy of meteors, Advances in Space Research, 33, 1444-1454, https://doi.org/10.1016/j.asr.2003.05.001, 2004.

Malhotra, A. and Mathews, J. D.: A statistical study of meteoroid fragmentation and differential ablation using the Resolute Bay Incoherent 
Mathews, J. D., Janches, D., Meisel, D. D., and Zhou, Q.-H.: The micrometeoroid mass flux into the upper atmosphere: Arecibo results and a comparison with prior estimates, Geophys. Res. Lett., 28, 1929-1932, https://doi.org/10.1029/2000GL012621, 2001.

McKinley, D. W. R.: Meteor Science and Engineering, McGraw-Hill Series in engineering sciences, McGraw-Hill Book Company, Incorporated, 1961.

Michell, R. G., DeLuca, M., Janches, D., Chen, R., and Samara, M.: Simultaneous optical and dual-frequency radar observations of small mass meteors at Arecibo, Planet. Space Sci., 166, 1-8, https://doi.org/10.1016/j.pss.2018.07.015, 2019.

Millman, P. M.: A general survey of meteor spectra, Smithsonian Contributions to Astrophysics, 7, 119, 1963.

Plane, J. M. C.: Cosmic dust in the earth's atmosphere, Chemical Society Reviews, 41, 6507-6518, https://doi.org/10.1039/C2CS35132C, 2012.

205 Rudawska, R., Tóth, J., Kalmančok, D., Zigo, P., and Matlovič, P.: Meteor spectra from AMOS video system, Planetary Space Science, 123, 25-32, https://doi.org/10.1016/j.pss.2015.11.018, 2016.

Sato, T., Nakamura, T., and Nishimura, K.: Orbit determination of meteors using the MU radar,, IEICE Trans. Commun., 2000.

Savage, H. F. and Boitnott, C. A.: Light Emission Measurements of Calcium and Magnesium at Simulated Meteor Conditions. I. CrossSection Measurements, The Astrophysical Journal, 167, 341, https://doi.org/10.1086/151036, 1971.

210 Sparks, J. J., Janches, D., Nicolls, M. J., and Heinselman, C.: Determination of physical and radiant meteor properties using PFISR interferometry measurements of head echoes, Journal of Atmospheric and Solar-Terrestrial Physics, 72, 1221-1230, https://doi.org/10.1016/j.jastp.2010.08.004, 2010.

Trigo-Rodriguez, J. M., Llorca, J., Borovicka, J., and Fabregat, J.: Chemical abundances determined from meteor spectra: I Ratios of the main chemical elements, Meteoritics and Planetary Science, 38, 1283-1294, https://doi.org/10.1111/j.1945-5100.2003.tb00313.x, 2003.

215 Vondrak, T., Plane, J. M. C., Broadley, S., and Janches, D.: A chemical model of meteoric ablation, Atmospheric Chemistry \& Physics, 8, 7015-7031, 2008.

Weryk, R. J. and Brown, P. G.: Simultaneous radar and video meteors-II: Photometry and ionisation, Planetary and Space Science, 81, 32-47, https://doi.org/10.1016/j.pss.2013.03.012, 2013. 